\title{
Metastatic Leiomyosarcoma of the Inferior Vena Cava Presenting with a Hepatic Mass
}

\author{
Ibrahem Adam R ${ }^{* 1}$, Patel $C^{2}$, Aggarwal SK ${ }^{1}$, McClluch $T^{3}$ and Kumaran $\mathrm{M}^{1}$ \\ ${ }^{1}$ Department of Radiology, Queen's Medical Centre, Nottingham University Hospitals NHS Trust, Derby Road, \\ Nottingham, United Kingdom \\ ${ }^{2}$ Department of Urology, Nottingham City Hospital, Hucknall Rd, Nottingham, United Kingdom \\ ${ }^{3}$ Department of Histopathology, Nottingham City Hospital, Hucknall Rd, Nottingham, United Kingdom
}

*Corresponding author: Ibrahem Adam R, Department of Radiology, Queen's Medical Centre, Nottingham University Hospitals NHS Trust, Derby Road, Nottingham, United Kingdom NG7 2UH, E-mail: renan. ibrahem@doctors.org.uk

Citation: Ibrahem Adam R, Patel C, Aggarwal SK, McClluch T, Kumaran M (2016) Metastatic Leiomyosarcoma of the Inferior Vena Cava Presenting with a Hepatic Mass. J Adv Radiol Med Image 1(1): 105. doi: 10.15744/2456-5504.1.105

Received Date: May 20, 2016 Accepted Date: July 25, 2016 Published Date: July 27, 2016

\begin{abstract}
A case report of a 72-year-old female patient presenting with right-sided abdominal pain and back pain, on which initial abdominal ultrasound revealed a hepatic mass. With the aid of subsequent CT imaging and biopsy, a leiomyosarcoma of the inferior vena cava was found. Palliative chemo-radiotherapy was offered to the patient. The literature on leiomyosarcoma, its presentation in the inferior vena cava and the use of diagnostic imaging has been discussed.
\end{abstract}

Keywords: IVC; Leiomyosarcoma; Hepatic mass; Sarcoma

\section{Introduction}

Leiomyosarcoma of the inferior vena cava (IVC) is the most common primary malignancy of the IVC. It is however a rare condition and makes up approximately $5-10 \%$ of all soft tissue sarcomas [1,2]. Complete surgical resection with negative margins is essential for long term survival $[1,3]$. We report this case to provide an educational and literature review of the clinical presentation, findings on imaging and management options.

\section{Case report}

A 72-year-old lady with a background of diet-controlled diabetes and hypertension, presented with 4 months history of nonspecific right-sided abdominal and back pain, which had not resolved with simple analgesia. On examination, she was found to have marked lower limb oedema extending up to the middle of the torso. Abdominal examination revealed palpable hepatomegaly, $1 \mathrm{~cm}$ below the sub-costal margin. There was no lymphadenopathy. Cardiovascular examination was normal and the jugular venous pressure (JVP) was not elevated. An initial ultrasound scan revealed a mass in the right lobe of the liver (Figure 1).

A CT scan revealed a heterogeneous soft tissue tumour originating from the inferior vena cava (IVC). The mass was centered on the intra-hepatic IVC measuring 46 x 61 x $122 \mathrm{~mm}$ extending down to the infra-hepatic IVC (Figure 2a). The IVC lumen was nearly fully occluded by the tumour. The radiological appearances were typical of an IVC leiomyosarcoma. The middle and right hepatic veins were occluded with radiological features in the right lobe of the liver similar to appearances of Budd Chiari syndrome (Figure 2b). CT imaging also demonstrated a nodule in segment 7 of the liver suspicious of a metastatic deposit alongside bilateral pulmonary metastasis and small volume ascites. The liver function tests and tumor markers including alpha-fetoprotein, carcinoembryonic antigen and CA19-9 were normal.

A transhepatic ultrasound-guided biopsy was performed. Histology showed a moderately pleomorphic spindle cell neoplasm with typical features of smooth muscle histogenesis (Figure 3). Positive immunohistochemical staining for smooth muscle actin and desmin confirmed a diagnosis of a leiomyosarcoma. Following discussion at the regional sarcoma MDT (multidisciplinary team) meeting a decision to treat the patient with palliative chemo-radiotherapy was made and the patient was not followed up further. 


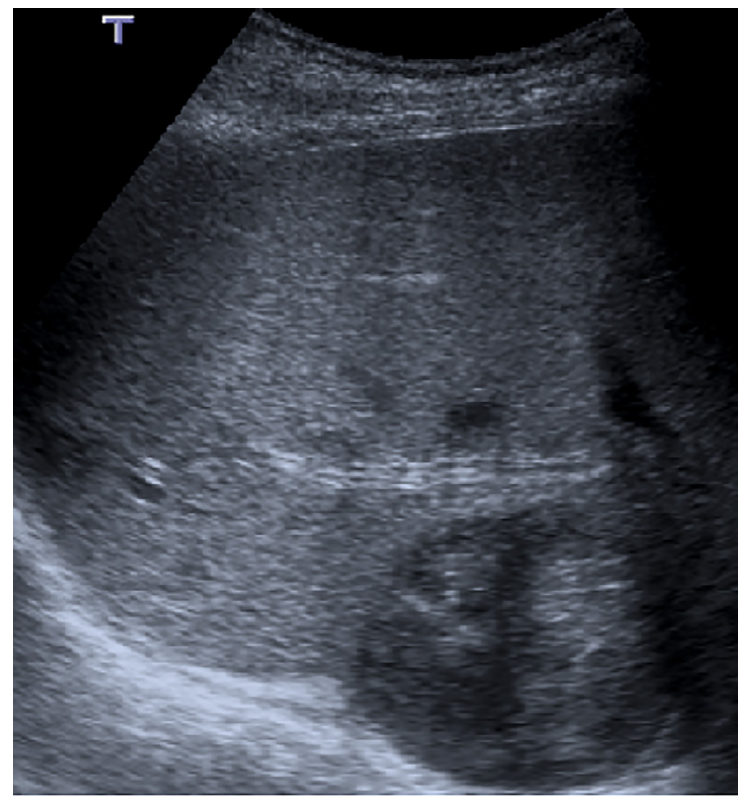

Figure 1: Ultrasound scan revealed a mass in the right lobe of the liver

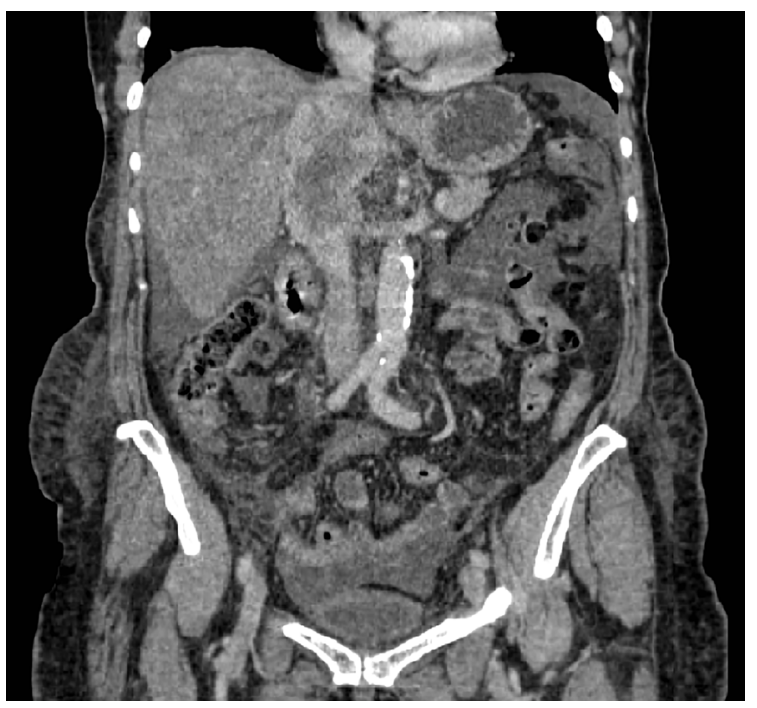

Figure 2a: Inferior Vena Cava measuring 46 x 61 x $122 \mathrm{~mm}$ extending down to the infra-hepatic IVC

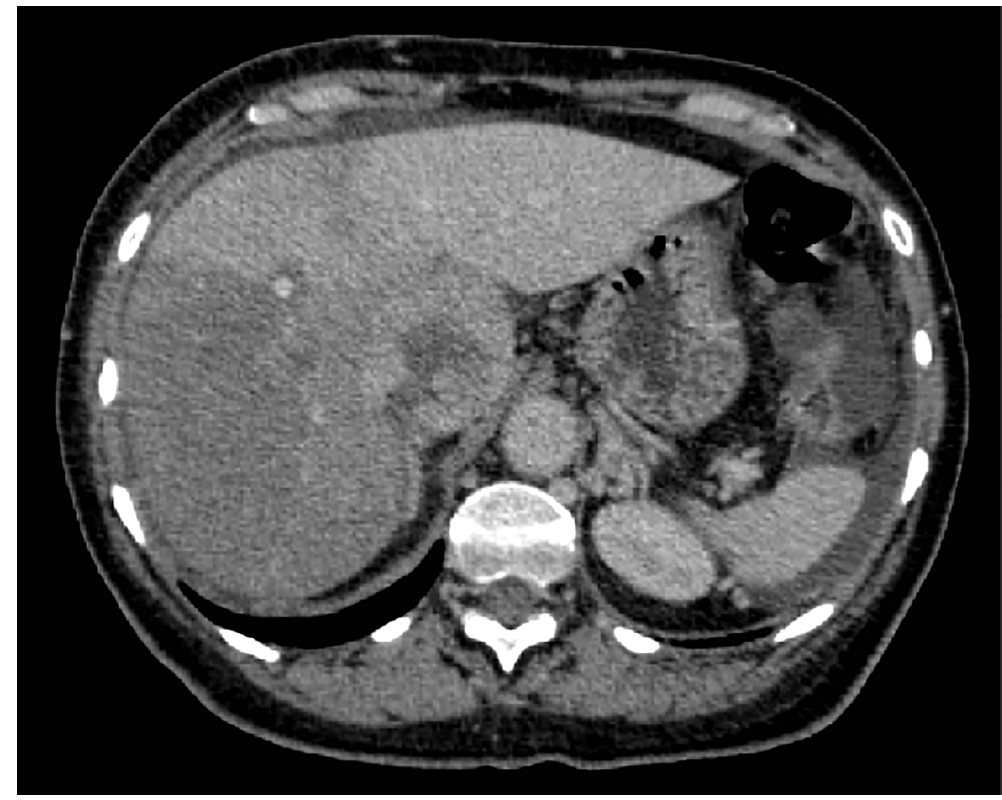

Figure 2b: Right lobe of the liver similar to Budd Chiari syndrome 


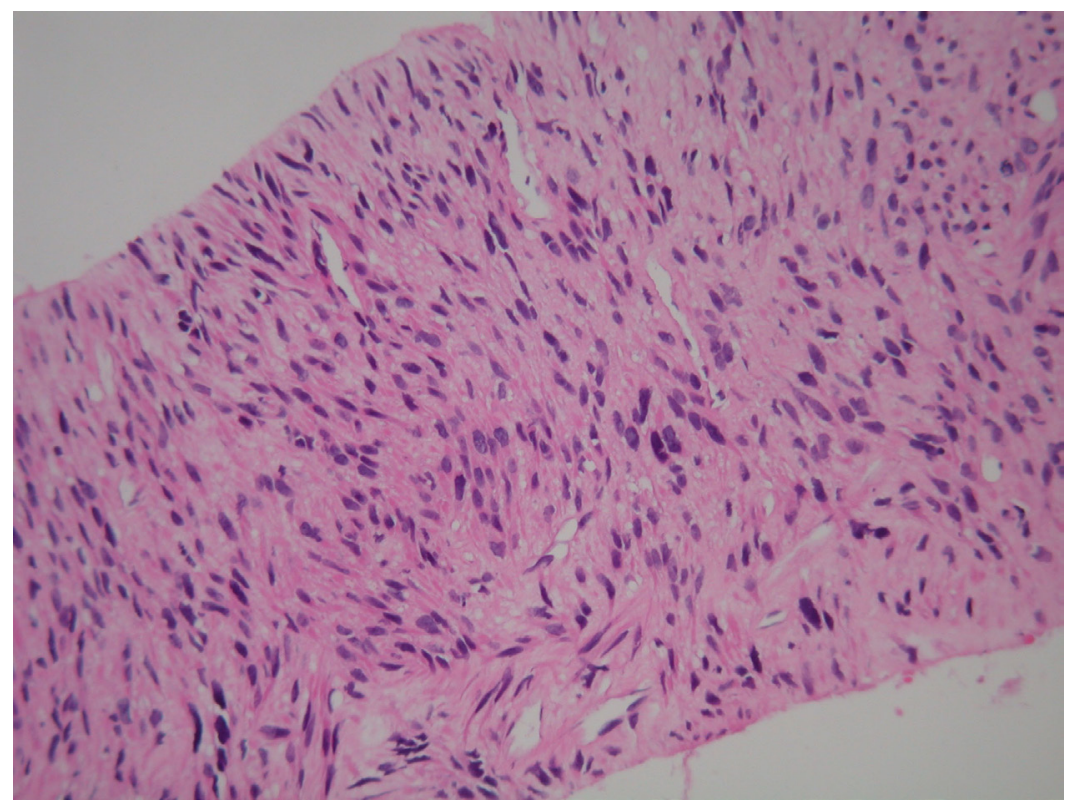

Figure 3: Histology showed a moderately pleomorphic spindle cell neoplasm

\section{Discussion}

Leiomyosarcoma (LMS) is a type of soft tissue sarcoma that originates from smooth muscle cells and considered the malignant counterpart of a leiomyoma. Leiomyosarcoma is considered to be the most common primary malignancy of the IVC; however a fewer than 300 cases have been reported. Its peak incidence is in the $6^{\text {th }}$ decade of life with predominance in women [4,5]. It is generally slow growing tumour and remains confined to the vessel wall in the early stages and patients can be asymptomatic for a long time prior to diagnosis. The presentation can vary dependent on the site of the tumour. Tumors that arise in the IVC infrahepatically present with lower extremity oedema and vague abdominal pain. If the tumor developed in the supra-hepatic segment of the IVC, Budd-Chiari syndrome can develop as a result of compression of the hepatic veins causing, hepatomegaly, jaundice, and ascites.

The aetiology of Leiomyosarcoma is not known, however there are genetic and environmental risk factors. Patients with Li Fraumeni syndrome are at greater risk of developing a leiomyosarcoma including IVC leiomyosacomas. Around 13\% children who have retinoblastoma develop a soft tissue sarcoma within 50 years. Radiotherapy treatment is thought to cause 3\% of leiomyosarcomas, usually 10-15 years after initial therapy. Environmental exposure to chemicals such as; vinyl chloride, dioxins and chlorophenols have also been linked. The risk of leiomyosarcoma along with other types of soft tissue sarcomas may be elevated in people with HIV, though this appears to be causatively linked to Epstein-Barr virus infection [6].

Kieffer, et al. has reported leiomyosarcomas of the inferior vena cava in one of the largest series of cases in 2006. Their results were in agreement with previous case reviews. Their main findings were: a female predilection, mean age in the sixth decile, abdominal pain as the presenting symptom, inconsistent oedema of the lower limbs, and potential for development of Budd-Chiari syndrome and intracardiac extension. They also studied the segment of the IVC involved in each case and found that segment II (interrenal and retrohepatic segment) was the most commonly involved segment and this raises challenges for operative treatment [7].

CT is the primary modality for assessment of suspected leiomyosarcoma and metastatic disease. Multiplanar capabilities of MRI are particularly useful to distinguish the precise tumour site and involvement of adjacent tissues. Gadolinium is used to assess tumour margins, vascularity and vessel involvement with MRI [8]. Tumours involving the large veins can often be intraluminal, extraluminal or a combination of both. These well-defined lesions may expand the vessel lumen and appear isointense to muscle on T1 and hyperintense on T2 weighted images [9].

Metastases from leiomyosarcomas spread haematogenously, with the liver and lungs being the most frequent sites of occurrence. Deep intra-abdominal, retroperitoneal and large vein tumours have the most frequent occurrence of metastases (40-50\%) [10].

Surveillance imaging varies; O'Sullivan, et al. report using contrast enhanced CT scan of the abdomen and pelvis every 6 months for 1 year and annually thereafter. CT thorax is used for surveillance in those with pulmonary metastasis at initial presentation and plain radiographs for those patients without any pulmonary metastasis initially [8].

Therapeutic procedures or intervention are dependent on the primary location of the tumour and the stage of the malignancy. Optimal treatment for leiomyosarcomas of the IVC could include complete resection of the malignant lesion with preservation of venous return to achieve long-term survival [3]. Following radical tumor resection, the IVC can be managed in various ways including; ligation, primary patch repair and expanded polytetrafluoroethylene (PTFE) tube grafting. 
Curative surgery of IVC leiomyosarcomas, in dedicated centers, can yield good functional results and acceptable survival rates, comparable to those of leiomyosarcomas in non caval veins $[11,12]$. When managing leiomyosarcomas it is important to obtain complete resection margins (R0 margin), in the vast majority of cases this requires extended resection of the venous collaterals, which leads to lower limb oedema. PTFE grafting is recommended to prevent lower limb oedema [11,12]. Adjuvant chemoradiotherapy has been used in these patients where there is absence of negative margin after resection, however the long term survical benefits are unclear clear with some authors reporting possible good survival and other non $[3,11,13]$.

Due to the rarity of leiomyosarcomas occurring in the IVC, the majority of data regarding treatment has come from small series and case reports. There is no developed standard or overall effective chemoradiotherapy regime which has shown to improve survival $[3,13]$.

The prognosis for leiomyosarcoma is relatively poor with an overall 5 year survival rate of $35 \%$. Hines, et al. studied the comparison of the 5 year cumulative survival rates of leiomyosarcomas of varying anatomical origins studying 14 cases. They found that the survival rates were $53 \%$ for patients with leiomyosarcoma of the IVC, $47 \%$ for those with leiomyosarcoma of the stomach, $43 \%$ for those with leiomyosarcoma of the small intestine, $56 \%$ for those with leiomyosarcoma of the retroperitoneum, and $65 \%$ for those with leiomyosarcoma of the uterus [13]. Hines, et al. concluded that despite having a tumor that originates from the IVC, patients with this tumor type can enjoy reasonably long term survival.

\section{Conclusion}

Leiomyosarcoma of the inferior vena cava can present with abdominal pain, lower limb oedema and Budd Chiari syndrome. CT is the primary modality for assessment of this malignancy. Optimal treatment for leiomyosarcomas of the IVC could include complete resection of the malignant lesion with preservation of venous return to achieve long-term survival. Adjuvant chemoradiotherapy can be offered to some patients however the benefits are still unclear.

\section{Author contributions}

IAR, PC, ASK, MT and KM prepared the manuscript. KM outlined the manuscripts layout and supervised the work. All Authors approved the final manuscript.

\section{References}

1. Imao T, Amano T, Takemae K ( 2011) Leiomyosarcoma of the renal vein. Int J Clin Oncol 16: 76-9.

2. Sokolich J, Mejia A, Cheng S, Dunn E (2008) Retroperitoneal leiomyosarcoma of the inferior vena cava mimicking a liver tumor. Am Surg 74: 433-6.

3. Dew J, Hansen K, Hammon J, McCoy T, Levine EA, et al. (2005) Leiomyosarcoma of the inferior vena cava: surgical management and clinical results. Am Surg 71: 497-501.

4. Drukker L, Alberton J, Reissman P (2012) Leiomyosarcoma of the inferior vena cava: radical surgery without vascular reconstruction. Vasc Endovascular Surg 46: 688-90.

5. Sessa B, Iannicelli E, Caterino S, D’Angelo F, Milione M, et al. (2010) Imaging of leiomyosarcoma of the inferior vena cava: comparison of 2 cases and review of the literature. Cancer Imaging 10: 80-4.

6. Bhatia K, Shiels MS, Berg A, Engels EA (2012) Sarcomas other than Kaposi sarcoma occurring in immunodeficiency: interpretations from a systematic literature review. Curr Opin Oncol 24: 537-46.

7. Kieffer E, Alaoui M, Piette JC, Cacoub P, Chiche L (2006) Leiomyosarcoma of the inferior vena cava: experience in 22 cases. Ann Surg $244: 289-95$.

8. O'Sullivan PJ, Harris AC, Munk PL (2008) Radiological imaging features of non-uterine leiomyosarcoma. Br J Radiol 81: 73-81.

9. Kransdorf M J, Murphy M D (1997) Imaging of soft tissue tumors. Philadelphia, WB Saunders. J Bone Joint Surg Am 79: 1277-8.

10. Khong PL, Chan GC, Shek TW, Tam PK, Chan FL (2002) Imaging of peripheral PNET: common and uncommon locations. Clin Radiol 57: $272-7$.

11. Illuminati G, Calio' FG, D’Urso A, Giacobbi D, Papaspyropoulos V, et al. (2006) Prosthetic replacement of the infrahepatic inferior vena cava for leiomyosarcoma. Arch Surg 141: 919-24.

12. Illuminati G, Pizzardi G, Calio' F, Pacilè MA, Masci F, et al. (2016) Outcome of inferior vena cava and noncaval venous leiomyosarcomas. Surgery 159: 613-20.

13. Hines OJ, Nelson S, Quinones-Baldrich WJ, Eilber FR (1999) Leiomyosarcoma of the inferior vena cava: prognosis and comparison with leiomyosarcoma of other anatomic sites. Cancer 85: 1077-83. 


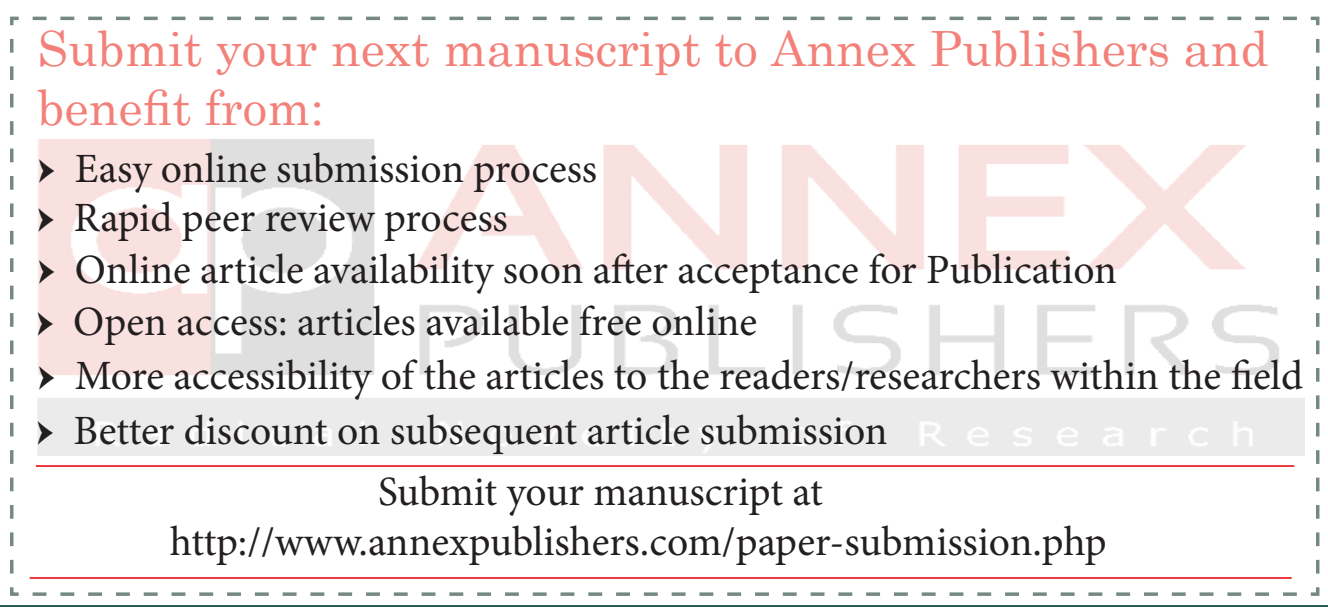

